\title{
Mesoscale Magnetism
}

\author{
Axel Hoffmann ${ }^{\mathrm{a}, *}$, Helmut Schultheiß ${ }^{\mathrm{b}}$ \\ ${ }^{a}$ Materials Science Division, Argonne National Laboratory, Argonne, Illinois 60439, USA \\ ${ }^{b}$ Institut für Ionenstrahlphysik und Materialforschung, Helmholtz-Zentrum Dresden-Rossendorf, D-01328 Dresden, Germany
}

\begin{abstract}
Magnetic interactions give rise to a surprising amount of complexity due to the fact that both static and dynamic magnetic properties are governed by competing short-range exchange interactions and long-range dipolar coupling. Even though the underlying dynamical equations are well established, the connection of magnetization dynamics to other degrees of freedom, such as optical excitations, charge and heat flow, or mechanical motion, make magnetism a mesoscale research problem that is still wide open for exploration. Synthesizing magnetic materials and heterostructures with tailored properties will allow to take advantage of magnetic interactions spanning many length-scales, which can be probed with advanced spectroscopy and microscopy and modeled with multi-scale simulations. This review highlights some of the current basic research topics in mesoscale magnetism, which beyond their fundamental science impact are also expected to influence applications ranging from information technologies to magnetism based energy conversion.
\end{abstract}

Keywords:

permanent magnet, magnetic soliton, spin texture, magnonics, optical magnetization switching, spin transfer torque, spin Hall effect, spin caloritronics, magnetomechanical coupling

\section{Introduction}

Magnetism is one of the quintessential examples of mesoscale behavior, which harnesses interactions between different degree of freedoms over a hierarchy of divergent lengthscales. Magnetic interactions span across a wide variety of length and time-scales - from ultrafast (fsec) exchange between neighboring atoms to long-range dipolar interactions, which enable probing and manipulating magnetism at a distance and at moderate speed. Concurrently, the electron spin can be coupled to charge transport and lattice vibrations through spin-orbit coupling, which generates complex pathways for controlling spin behavior. For these reasons magnetism is key to many modern applications, ranging from information technologies to energy conversion. For example, with respect to information technologies, the grand challenge for progressing towards the thermodynamic Landauer limit of information processing [1] is the conundrum of combining data retention, demanding high thermal stability, with low-power computation, requiring small energy differences. Towards this end, modern magnetic materials and devices contribute significantly to improvements of computational performance and energy efficiency of both data storage and logic. For energy applications the important issues are the inherent energy stored in the magnetization and the interaction with charge and heat currents. New surprising solutions for many of these applied problems come from recent basic science developments, such as spin currents, spin torques, elec-

\footnotetext{
${ }^{*}$ Corresponding author

Email addresses: hoffmann@anl.gov (Axel Hoffmann), h.schultheiss@hzdr.de (Helmut Schultheiß)
}

tric field or photonic effects. Similarly, the ability to synthesize emerging magnetic materials and heterostructures with tailored properties, which take advantage of the magnetic interactions across various fundamental length- and time-scales, will enable to harness the full potential of these new physical phenomena.

The main focus of current magnetism research revolves around fundamental science issues involving competing interactions. This encompasses static and dynamic magnetization textures, which balance short-range exchange with long-range dipolar interactions and give rise to critical phenomena, such as magnetic phase transitions. Similarly, interactions at materials interfaces and surfaces can result in completely new spin phenomena, such as emergent magnetism at complex oxide interfaces, hard/soft core shell structures or the quantum spin Hall effect, when Rashba spin-orbit coupling can overcome the bulk bandgap in topological insulators [2]. This enables fundamentally new energy efficient ways of coupling spin transport to other degrees of freedom, including electric fields or charge and heat transport. Recent examples include novel pathways to multiferroic behavior from rotational degrees of freedom [3], unexpected coupling between spin and heat currents in ferromagnetic insulators [4], and the switching of magnetization via direct charge currents and spin-orbit coupling [5, 6]. The latter has direct applied impact, since it eliminates the need for a magnetic reference layer for spin torque effects, which in turn enables novel schemes of magnetic information technologies with the ultimate goal of controlling spin properties down to the single-spin level. This research requires the combined effort of cutting edge synthesis, such as deposition and self-assembly methods, analytical tools such as X-ray, neutron, electron, and 
probe-based spectroscopies and microscopies, ultrafast X-ray and optical techniques as well as transport and magnetometry measurements; and theoretical efforts in the computation of the electronic structure of these materials and in modeling magnetic distributions, domain distributions and dynamics and transport properties.

Clearly the scope of mesoscale magnetism is too vast to be reviewed comprehensively within this brief review. Instead we focus on several examples of current research topics, which highlight some of the common themes of competing interactions over different length scales and coupled degrees of freedom.

\section{Competing length and time scale}

Interactions across many length and time scales are ubiquitous in magnetism. They range from inter-atomic exchange coupling, which results in ultrafast (fs) dynamics to long-range dipolar interactions, which everybody who ever played with magnets is familiar with, resulting in domain structures on macroscopic scales and magnetization dynamics as slow as seconds and minutes. In between there are a whole range of additional interactions, such as magnetic anisotropies, spin-orbit coupling, Zeeman interactions with external magnetic field, etc. The competition between these various interactions result in a rich variety of spatial and temporal spin textures, some of which are discussed in more detail in the following sections.

\subsection{Exchange springs}

The practical use of magnetic materials started with the use of permanent magnets as compass needles around a thousand years ago and even today the permanent magnetization is a key component in many energy conversion technologies [7]. A key measure for the effectiveness of permanent magnets is the energy product $(B H)_{\max }$, and its optimization typically requires high coercivity and large remanent magnetization. Unfortunately, the later two properties are generally inversely correlated; namely magnetic hard materials with high anisotropy (and thus high coercivity) tend to have low magnetic moments, while materials with large magnetization in turn tend to be soft, i.e., have low anisotropy. One pathway out of this dilemma is the concept of an exchange spring magnet, which was first introduced by Kneller and Hawig [8]. The basic principle is to combine hard and soft magnetic materials in composite materials and harness the best of both properties through interfacial exchange coupling. The optimization of such a composite structure is non-trivial, since it combines the exchange and anisotropy interactions on an atomic scale, which result in short-range magnetization changes in the hard-phase and longrange magnetization changes in the soft phase [9]. Furthermore, very long-range magnetostatic interactions may become relevant for the exact magnetization reversal starting in the softphase. This complexity is illustrated in Fig. 1, which shows the maximum possible energy product for hard-soft composites with different morphology and different soft-phase volume fraction. Experimentally, most systematic studies have focused on (a)
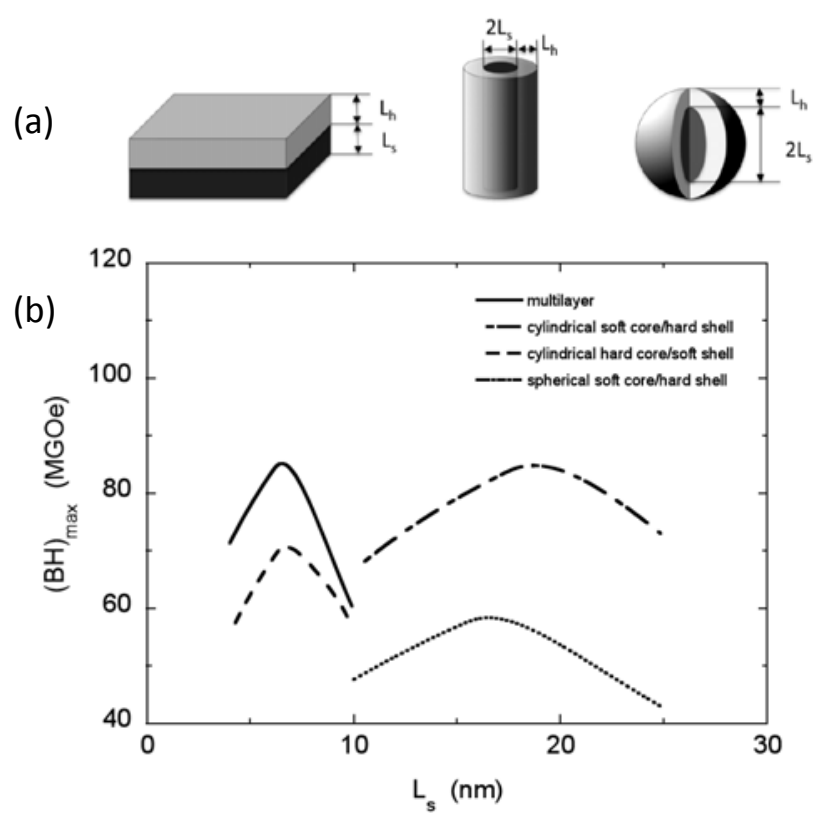

Figure 1: (a) Schematic depiction of different possible geometries for exchange spring nano-composites based on multilayers, cylindrical and spherical core/shell structures. (b) The maximum energy product $(B H)_{\max }$ as a function of the soft phase size $L_{s}$ for different geometries. Reprinted figure with permission from Ref. [12], (C) 2014 IOP Publishing Ltd.

multilayered systems [10], while core-shell spherical particles are preferred due to their scalability to large-scale production [11]. However, as is evident from Fig. 1 a cylindrical softcore/hard-shell structure is preferable due to its large energy product and its relaxed requirements for size control. Interestingly, in these cylindrical exchange spring structures, the spin texture during the partial reversal of the soft phase resembles a magnetic skyrmion structure [see Fig. 2(b)], and the associated change in topology is related to the relative high nucleation field. Future work can be expected to focus on experimentally verifying these theoretical predictions, as well as, designing efficient fabrication processes for novel composite permanent magnets.

\subsection{Magnetic solitons}

The competition between different magnetic interactions, most importantly exchange interactions at the atomic scale and long-range magneto static coupling, gives rise to a wide range of magnetic topological solitons [13]. These can exhibit quasiparticle-like behavior with their own well defined dynamics, which can couple to external magnetic fields and electric currents. One of the simplest example is a magnetic domain wall, which forms between magnetic domains with different magnetization directions. The discovery that such domain walls can be moved with electric currents [14] has inspired the concept of using these domain walls for encoding information in solid-state memories [15]. The physics of domain walls and their manipulation is surprisingly rich and exemplifies the complexities that emerge in mesoscale problems. For example, the 
internal degrees of freedom of a domain wall may give rise to complex dynamics at a variety of time scales [16].

Similar multi-scale behavior is also observed for other magnetic topological solitons. Of these, magnetic vortices [17, 18] have been studied recently in great detail. Magnetic vortices are stabilized by magneto static interactions and they form in planar magnetic structures as an in-plane curling magnetization (resulting in two possibility chiralities) with a central singularity, the vortex core, which points perpendicular to the plane (and thus two possible polarities). The vortex core has a diameter of a few nm due to the relative short range of the exchange energy [19], but stabilizing magnetic vortices requires a minimum size of the magnetic element of at least an order of magnitude larger [20]. Furthermore, these spin structures can be transferred via interfacial exchange coupling into other materials, such as antiferromagnets, which on their own would not develop a magnetic vortex structure [21, 22].

Magnetic vortices have well-defined gyrotropic resonant motions in the sub-GHz frequency range [23, 24]. At the same time the behavior of individual vortices can be altered in a controlled way through coupling to other vortices, either via dipolar coupling for separated structures or direct exchange in touching vortices [25]. This offers the exciting possibility to use magnetic vortices as individual building blocks for more complex structures and thereby explore the hallmark of mesocscale behavior: namely the evolution from individual to collective behavior. In that sense, one can envision building complex magnonic crystals from arrays of vortices, with the possibility of tailoring the resultant band structure of the magnetization dynamics through the high degeneracy of magnetic configurations, since each vortex may support four different equilibrium configurations (combinations of the two polarities and two chiralities). In fact, a first step in this direction has been recently realized by showing that the magnetization configuration of two interacting vortices can be deterministically controlled through targeted dynamic excitations [26]. This opens the door towards developing fully programmable magnetic matter, where the dynamic properties become reconfigurable, similar to quantum cellular automata $[1,27,28]$. An important necessary development towards the detailed understanding of these coupled magnetic systems will be the development of tools that allow to quantify the interactions carefully [29].

Interestingly, magnetic vortices form half of another type of a magnetic topological structure, which received recently increased attention: magnetic skyrmions. They were first discovered via neutron scattering in helical magnetic materials with chiral interactions [33]. What sets skyrmions apart from the previously discussed solitons is the fact that the spins of magnetic skyrmions point in all possible three-dimensional directions. Figure 2(a) shows schematically how a skyrmion spin structure can be derived by unfolding a hedgehog, i.e., a sphere with spins perpendicular to its surface. Although skyrmions were first discovered in materials with chiral interactions, where they form regular lattices as depicted in Fig. 2(b), these chiral interactions are not necessary for their formation [34]. In fact, magnetostatic interactions in thin films with perpendicular anisotropy can stabilize so-called magnetic bubbles, which can

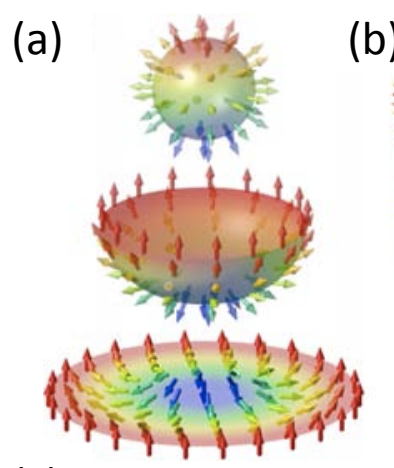

(b)
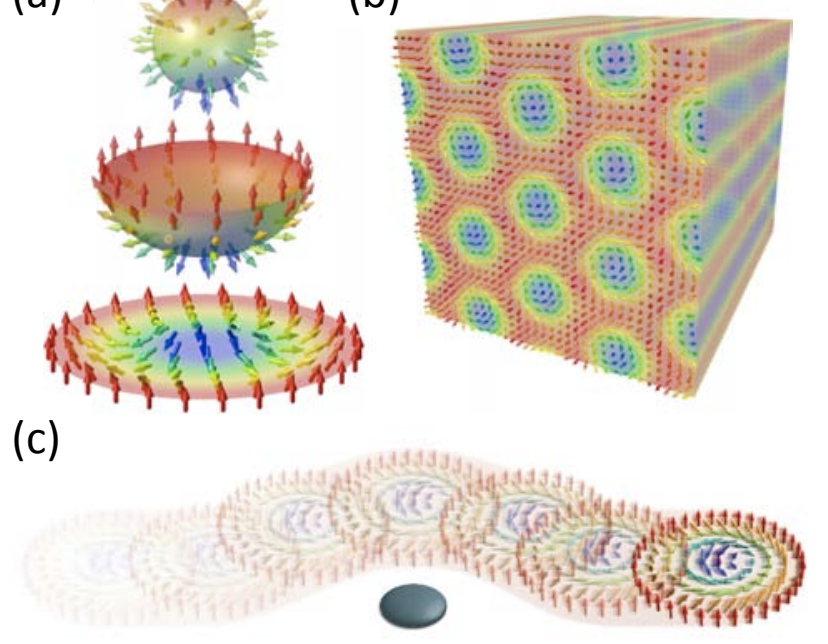

(d)

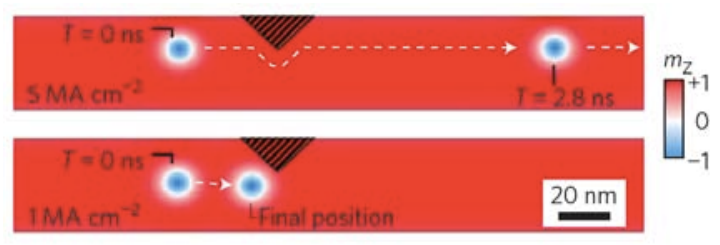

Figure 2: (a) Schematic showing how a skyrmion spin configuration is obtained from "unfolding" a hedgehog. (b) Representation of a skyrmion lattice. (c) Schematic showing how a skyrmion can move around an obstacle, resulting in weak pinning. (d) Micromagnetic simulation of current-induced skyrmion motion in confined geometries with pinning simulated by enhanced anisotropy. Reprinted figures with permission from: (a),(b) Ref. [30] (c) 2014 AIP Publishing LLC,(c), (d) Refs. [31, 32] (C) 2013 Macmillan Publishers Ltd: Nature Nanotechnolgy.

be topologically equivalent to the skyrmions in helical magnets $[35,36]$. At the same time, combining thin ferromagnetic films with layers of heavy elements with strong spin-orbit coupling can give rise to chiral interfacial interactions [37, 38], stabilizing a skyrmion spin structure for magnetic bubbles, which can exist over a wide range of length scales. One surprising consequence of the skyrmion spin structure is that the related real space Berry phase of the spin texture gives rise to virtual magnetic fields affecting the electron motion and resulting in topological Hall effects in the absence of external magnetic field $[39,30]$. These virtual magnetic fields scale inversely with the skyrmion size and can reach values of 10,000 $\mathrm{T}$ for atomically sized skyrmions [40].

The high current interest in skyrmions arises from the fact that they can be manipulated very efficiently with electric currents with ultra-low threshold current densities [41]. One of the reasons is that due to their two-dimensional structure, skyrmions can move around pinning centers as is shown schematically in Fig. 2(c). In fact, such behavior was shown explicitly by micro magnetic simulations [32] as shown in Fig. 2(d), where the skyrmion moves around an artificial pinning center even for moderate current densities, much lower than what is normally required for current induced domain wall motion in wires. At the same time it was shown with 
spin-polarized scanning tunneling microscopy that spin polarized electric currents can be used for selectively creating and annihilating individual metastable skyrmions [42]. Therefore it is conceivable that topological charges based on magnetic skyrmions may replace the electronic charges used in current day information technologies. Especially the low dissipation manipulation of magnetic skyrmions may enable overcoming the heat generation bottleneck faced by modern semiconducting electronics. Towards this end typical mesoscale problems, such as how confined geometries influence skyrmion behavior, will need to be addressed.

\subsection{Magnonics}

Spin waves, also referred to as magnons indicating their quasi-particle character, are the excitation quanta of the spin system in a magnetic condensed matter body. They are collective excitations of the electron system and can be understood as a coherent precession of the electrons spins where the phase difference of neighboring precessing spins determines the spinwave wavelength. The collective nature of spin waves is established by short-range exchange interaction as well as nonlocal magnetic dipolar interaction, resulting in coherence of spin waves from mesoscopic to even macroscopic length scales. As one consequence of this collective interaction, spin waves are charge current free and, therefore, less subject to dissipation caused by scattering with impurities on the atomic level. This renders spin waves a promising candidate for the transport of spin information - one of the grand challenges of condensed matter physics. The possibilities of spin waves being a new means of information carrier stimulated the emerging research field called magnonics [43, 44, 45]. Its name - inspired by the terms spintronics and photonics - indicates the main driving force behind magnonics: exploiting spin waves for information processing. For frequencies ranging from gigahertz to terahertz, the spin-wave wavelengths can be as small as only a few nanometers, orders of magnitude smaller compared to electromagnetic waves.

The first building blocks for realizing a magnonic logic are already experimentally realized by modulating the phase of spin waves propagating in yttrium iron garnet waveguides [46]. This is possible since the spin-wave dispersion is strongly anisotropic and highly sensitive to varying magnetic fields. Hence, for a given frequency the spin-wave wavelength significantly depends on the relative angle between their wavevector and the magnetization direction as well as the magnitude of the internal magnetic field. This not only imposes challenges on spin-wave transport on small length scales but also brings about advantages for systematically manipulating spin waves. For example, locally applied magnetic fields can control the wavevector of spin-wave propagation [47, 48]. Furthermore, in the linear regime, the anisotropy leads to the formation of spin-wave caustics, which are non-diffractive spin-wave beams with a diameter smaller than the wavelength $[49,50]$. In magnonic crystals, the periodic modulation of the magnetic properties either in one or two dimensions allows to redesign the spin-wave dispersion in order to artificially create band gaps in which propagation is prohibited $[51,52,53]$. And due to the sensitivity of the

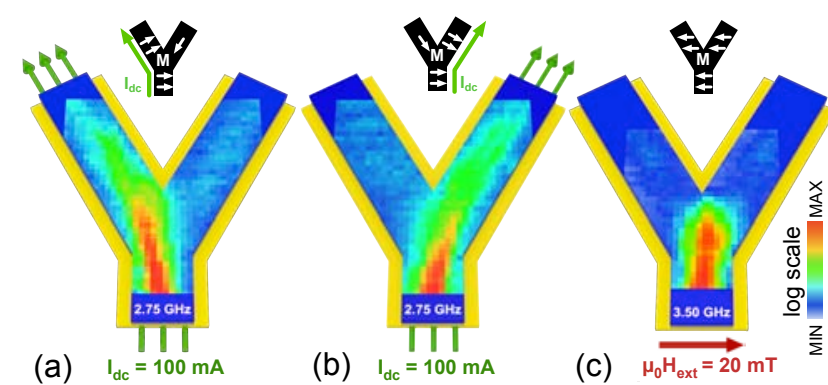

Figure 3: Demonstration of a spin wave multiplexer. The spin wave amplitude is imaged using Brillouin light scattering microscopy. Using Oersted magnetic fields from currents applied in different directions of the device allows to guide spin-waves into the left (a) or right (b) arm at the Y junction. Using a global homogenous field (c) results in spin waves stopping at the junction. Reprinted figure with permission from: Ref. [58] (c) 2014 Macmillan Publishers Ltd: Nature Communications.

spin wave dispersion to changes in the saturation magnetization it is even possible to imprint magnonic crystals and waveguides into a continuous ferromagnetic film without changing the topography by using local ion implantation [54, 55]. Once the magnetization is driven far from equilibrium, the intrinsic nonlinearity of the spin-wave dispersion causes a transformation of propagating spin-wave packets into solitons [56] which also opens the door for chaos and self-organization [57].

The fact that the magnetization orientation, which is nonvolatile and still reconfigurable on a sub-nanosecond timescale [59], imposes an anisotropy on spin waves due to long-range, magnetic dipolar interaction, can also be used to build reprogrammable magnonic crystals [60] or even a spin-wave multiplexer [58] as shown in Fig. 3. The multiplexer employs local magnetic fields generated by electric currents to magnetize parts of a Y-shaped microstructure transverse to the spin-wave propagation direction and, therefore, switches the anisotropy direction in order to actively steer spin waves. But besides the external control of the magnetization direction by magnetic fields one can make use of the topological solitons mentioned in section 2.2. Magnetic domain walls are well known to be a possible candidate for manipulating spin waves on short length scales due to their inherent inhomogeneous magnetization configuration $[61,62]$ or, in the reversed process, to be manipulated by spin waves [63] due to transfer of angular momentum. Even the switching of the core polarity of magnetic vortices can be triggered by spin waves [64] and we are looking forward to see experiments on the interaction between skyrmions and magnons [65]. The non-volatile and reprogrammable character of magnetic solitons make them especially interesting for applications in magnonic devices. However, their interaction with spin waves is also of fundamental physical interest regarding the questions of angular momentum transfer resulting in the displacement and the excitation of dynamics of these topologically protected objects.

The true mesoscale potential of magnonics lies within the vast range of possible interactions starting from the coupling between spin waves and magnetic solitons within the spin system but also going beyond by exploiting the coupling with elec- 
tric (spin) currents on one side and the coupling to photons via magneto-optical effects on the other side. Thus, magnonics is becoming a powerful bridge technology closing the gap on the time- and length-scale between charge and photon physics. On the one side, the direct and inverse spin Hall effects as well as spin pumping and spin-transfer torque, which we will discuss in section 3.2, allow for the mutual conversion of spin waves in spin and electric currents and vice versa. On the other side, novel experiments regarding the all-optical switching of magnetization and the manipulation of surface plasmons in magnetic thin films, see section 3.1, promise a wealth of possible interactions between photons and spin waves.

\subsection{Ultrafast dynamics}

The phenomena in the discussion on competing length and time scale, so far, were getting faster and faster. In the first section we discussed novel materials for permanent magnets, which are static, i.e., essentially no time dependence. The dynamics of magnetic solitons such as domain walls and vortices have eigenfrequencies ranging up to hundreds of Megahertz which means they are living on the time scale of a few nanoseconds. In magnonics the relevant time scale is given by the spin-wave frequencies, which are typically ranging from the Gigahertz to the lower Terahertz regime, i.e., on the picosecond timescale. In this last part of section 2 we want to discuss the ultrafast dynamics of ferromagnets, which happen when the system is removed from equilibrium by an intense, femtosecond laser pulse. The fundamental question addressed in these experiments is: How fast can the magnetization in a ferromagnet be destroyed and where does the angular momentum go? These experiments were made possible by the development of laser amplifier systems providing intense laser pulses that are only a few femtoseconds long. In a pioneering work Beaurepaire and coworkers used such a laser system to heat up the electron system and to probe the state of the magnetization with a second, time-delayed laser pulse via the magneto-optical Kerr effect with a temporal resolution limited only by the duration of the laser pulse [66]. The surprising result of this and following experiments was, that the magnetization of various ferromagnets - elemental and compounds - can be destroyed within fractions of a picosecond. Even though there was a very fast agreement that the demagnetization happens that fast, the latter part of the initial question - where the angular momentum is going during and after the demagnetization - is still an ongoing debate [67, 68].

An intriguing experiment to address this question was recently done by Rudolf et al. [69] by studying the demagnetization of a Ni-Fe multilayer stack. Both elements on their own show different demagnetization times, Ni being faster than $\mathrm{Fe}$. A pump-probe experiment using soft x-rays [shown in Fig. 4(a)] allows for element-selective measurements by tuning the $\mathrm{x}$-rays to a specific absorption edge. Using this approach they measured the demagnetization of the $\mathrm{Ni}$ and $\mathrm{Fe}$ layer for parallel and anti-parallel alignment of the magnetic moments. Surprisingly, for parallel alignment the magnetization of the Fe layer is increasing with time [see Fig. 4(b), red circles]. This can be understood by a superdiffusive spin current originating from (a)

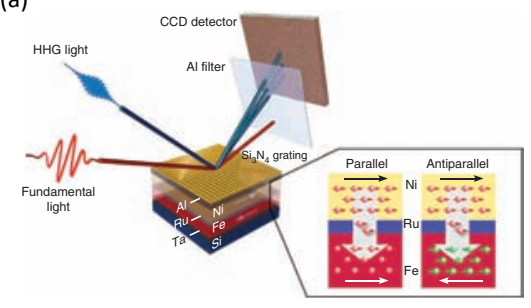

(b)

(c)

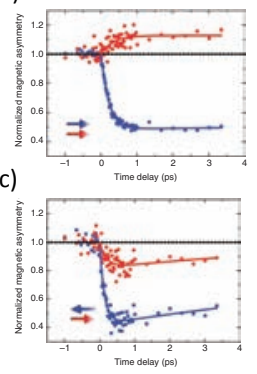

Figure 4: (a) Experimental setup for studying the ultrafast magnetization dynamics using a fs laser pulse for excitation (fundamental light, red) and the higher harmonics in the XUV range (HHG light, blue) for probing the magnetization. The wavelength of the higher harmonics is tunable in a wide range allowing for an element-selective detection of the magneto-optical activity in the multilayer stack. (b) and (c) show the demagnetization curves for $\mathrm{Ni}$ (blue) and $\mathrm{Fe}$ (red) as a function of time for parallel and antiparallel alignment of the initial magnetization directions in the multilayer. Reprinted figure with permission from: Ref. [69] (c) 2012 Macmillan Publishers Ltd: Nature Communications.

the Ni layer, which is demagnetizing faster than the Fe layer and actually shows a decrease in magnetization over time. The superdiffusive spin-current generated by the ultrafast demagnetization using femtosecond laser pulses can even generate $\mathrm{THz}$ radiation as shown by Kampfrath et al. [70]. Nevertheless, as pointed out by Koopmans et al. [68] the actual mechanism for ultrafast demagnetization depends on the material system: for itinerant ferromagnets like $\mathrm{Co}$ and $\mathrm{Ni}$ the underlying physics differs from ferromagnets with localized magnetic moments such as $\mathrm{Ga}$ and other $3 \mathrm{~d}$ transition metals. But without any doubts, the research based on optical stimulation of ferromagnets triggered a series of new experiments such as all-optical switching, which is discussed in section 3.1, and the excitation of spin waves using ultrashort laser pulses [71, 72].

\section{Magnetic manipulation beyond magnetic fields}

The manipulation of magnetization structures is of course most obvious through the application of external magnetic fields. Nevertheless, recently there has been increased interest in alternative ways of controlling magnetic states through optical excitations, electric and heat currents, and directly through mechanical motion. In this section we will discuss various recent developments that also highlight how a detailed understanding of phenomena at the mesoscale is required for a comprehensive investigation of their underlying physics.

\subsection{Optical}

As discussed in section 2.4 optical techniques allow to investigate dynamics at ultrafast time scales. It came as a surprise in 2007 that ultrafast laser pulses can deterministically switch magnetization directions in ferrimagnets, with the final magnetization solely determined by the helicity of the circular polarized light pulse [73]. The initial measurements were performed on rare earth/transition metal alloys, which led to the suggestion that different spin dynamics with different time 

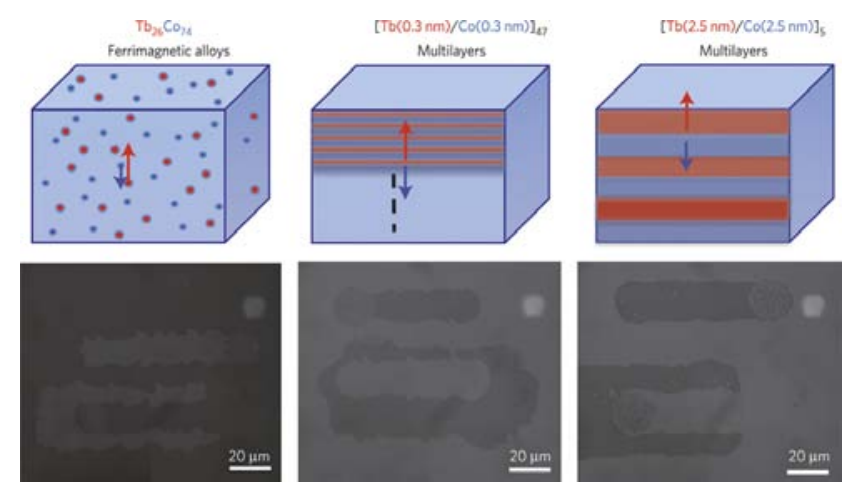

Figure 5: Optical helicity-dependent switching of ferrimagnetic materials with identical average composition: A random $\mathrm{Tb}_{26} \mathrm{Co}_{74}$ alloy and two superlattices with equal thickness Tb and Co layers, but different modulation lengths of 0.6 $\mathrm{nm}$ and $5 \mathrm{~nm}$, respectively. Reprinted figure with permission from: Ref. [74] (C) 2014 Macmillan Publishers Ltd: Nature Materials.

scales for the rare earth and transition metal spins are responsible for the helicity dependent magnetization response. More recently, it has been shown that this helicity dependent response can be observed in a wider class of ferrimagnetic systems [74]. As shown in Fig. 5 a random $\mathrm{Tb}_{26} \mathrm{Co}_{74}$ alloy shows the same helicity-dependent switching, as multilayers with the same average composition but different multilayer composition. What all these samples have in common, is that they have a different temperature dependence for the magnetization of the rare earth elements and the transition metal elements, leading to a compensation temperature above room temperature, at which both sub lattice magnetizations are equivalent. In fact, using this idea Mangin et al. showed that helicity dependent optical switching can also be observed in synthetic ferrimagnetic systems, consisting of only transition metal layers antiferromagnetically coupled through interlayer exchange coupling with thin Ir layers [74]. The situation became even more complex with the recent observation of all-optical helicity dependent switching in ferromagnetic systems [75], where it was demonstrated that this mechanism is even applicable to current advanced magnetic recording media. It appears that the general ingredients for understanding this phenomenon includes heating close to $T_{c}$ (or the compensation temperature for ferrimagnets), at which point the magnetic field or the angular momentum transfer from the laser beam (e.g., the inverse Faraday effect) is sufficient to set a preferred magnetization direction. During the subsequent cooldown it is then also important to engineer the material such that magneto static interactions do not give rise to significant domain formation. But it is also conceivable that more complex interactions between photo-excited conduction electrons and local spins matter in order to solve the puzzle of this alloptical magnetization switching.

The reverse mechanism of manipulating light on mesoscopic length scales using magnetism was recently demonstrated by Temnov et al. [76]. The experiment, which is outlined in Figure 6 , involves surface plasmon polaritons propagating in a $\mathrm{Au} / \mathrm{Co} / \mathrm{Au}$ multilayer. Plasmon polaritons are hybridized excitations of electrons and electromagnetic waves in a metal at
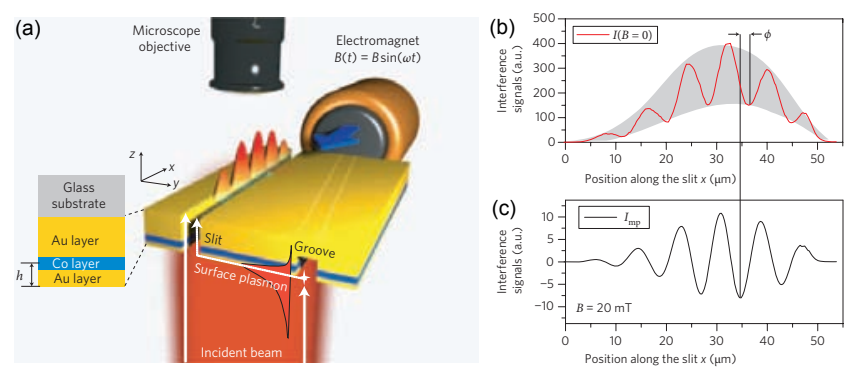

Figure 6: (a) Excitation and detection of surface plasmons in a $\mathrm{Au} / \mathrm{Co} / \mathrm{Au}$ film. Plasmons are excited by a laser at the grove and propagate in the film to the slit. There the optical energy is radiated into free space and interferes with the original laser light. The interference pattern reveals the phase of the plasmon. (b) and (c) interference pattern with and without magnetic field revealing the phase difference $\phi$ caused by the magneto-optical interaction between the plasmon and the Co layer. Reprinted figure with permission from: Ref. [76] (c) 2010 Macmillan Publishers Ltd: Nature Photonics.

optical frequencies. One intriguing feature is that they can confine optical energy in a volume smaller than their wavelength making them very attractive for photonic applications. The measurements revealed that the phase of a propagating surface plasmon polariton can be modulated on short length scales by reversing the direction of the magnetization in the Co film due to the strong magneto-optical coupling. This result was recently transferred to single nano magnets made from $\mathrm{Ni}$, which showed, that the presence of localized plasmon resonances have a dramatic impact on the magneto-optical activity of these nanomagnets [77]. This strong interaction between magnetism and plasmonics and the potential implications of this modulation of light on the nanometer scale combined with magnetization dynamics in the $\mathrm{GHz}$ to $\mathrm{THz}$ frequency range will tie the knot between magnonics and nano-photonics.

\subsection{Electric current}

Electric manipulations of magnetization through spintransfer torques was first theoretically predicted independently by Slonczewski [78, 79] and Berger [80], and subsequently experimentally detected as current-induced magnetization switching [81] and magnetization dynamics [82, 83]. In these early experiments the spin-transfer torque was demonstrated by passing the electric current first through a ferromagnetic conducting layer, which generated a spin-polarized charge current, whose angular moment can be absorbed by a second ferromagnetic conducting layer. This general principle is applicable to all metallic systems, tunnel junctions, and even single magnetic layers with inhomogeneous magnetization structures. In the later case, regions with different magnetization directions and domain walls in between them play a similar role as the individual layers in magnetic heterostructures with the distinction that now the spin transfer torque may result in a movement of the domain wall [14, 15, 37, 38, 41].

More recently, the need for an initial spin-polarizing metallic ferromagnet has been removed by using strong spin orbit coupling in nonmagnetic conductors. The spin-orbit coupling can result in spin currents transverse to the charge current, a phenomenon known as spin Hall effect [84, 85, 86]. Alternatively 

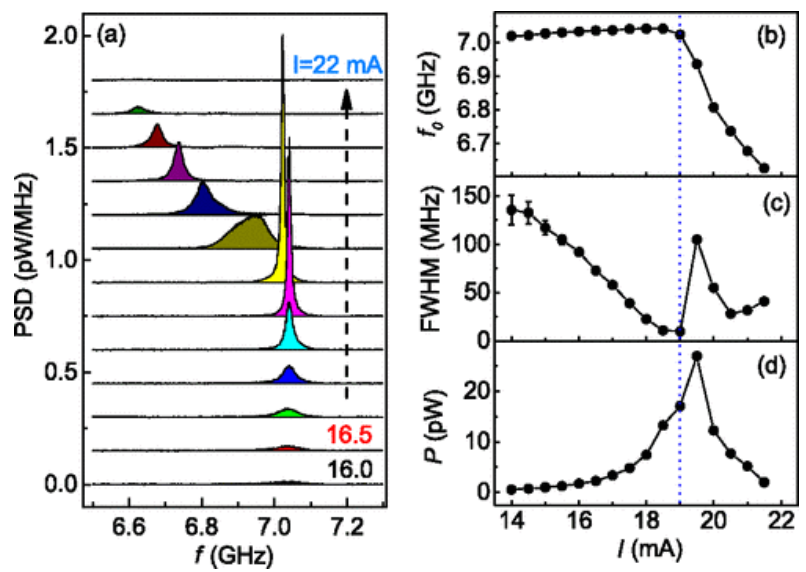

(e)

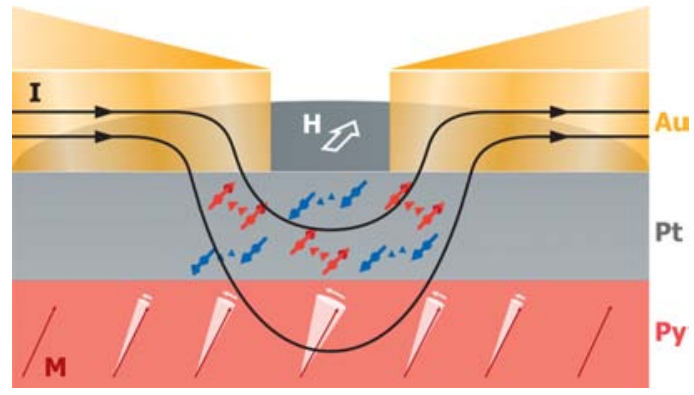

Figure 7: Current dependence of magnetization dynamics generated in a permalloy/Pt disk. (a) Spectra for different $d c$ currents. Current dependence of (b) central generation frequency, (c) line-width, and (d) integrated power. (e) Schematic of the current distribution, spin current generated in Pt and magnetization dynamics in permalloy. Reprinted figure with permission from: (a)-(d) Ref. [91] and (e) Ref. [94] (c) 2013 American Physical Society.

direct current-induced spin polarizations can occur in systems with reduced symmetries (e.g., interfaces), which is known as the Edelstein effect [87]. These effects are most pronounced for materials that contain heavy elements, where the spin-orbit coupling can even result in fully spin-polarized surface states, i.e., in topological insulators [2]. The spin-transfer torque from these spin-orbit coupling effects have been shown to be sufficient for magnetization switching $[5,6,88]$ and excitation of magnetization dynamics [89, 90,91]. One distinct advantage of using spin-orbit coupling as the source of spin currents is that it allows for spin-transfer torques with insulating materials [92, 93], where direct electrical injection is impossible.

The full mesoscale complexity of magnetization dynamics driven by spin-orbit interactions is illustrated by the spin Hall oscillator shown in Fig. 7(e). The basic underlying idea is that passing a $d c$ current through a bilayer of platinum and permalloy $\left(\mathrm{Ni}_{80} \mathrm{Fe}_{20}\right)$ the current in the Pt layer will generate via the spin Hall effect a spin accumulation at the interface with permalloy, which in turn can reduce or enhance the damping of the magnetization dynamics in permalloy through spin-transfer torque. Interestingly, for $d c$ currents flowing homogeneously through extended bilayer samples the damping cannot be reduced to the point where auto-oscillations are induced due to a
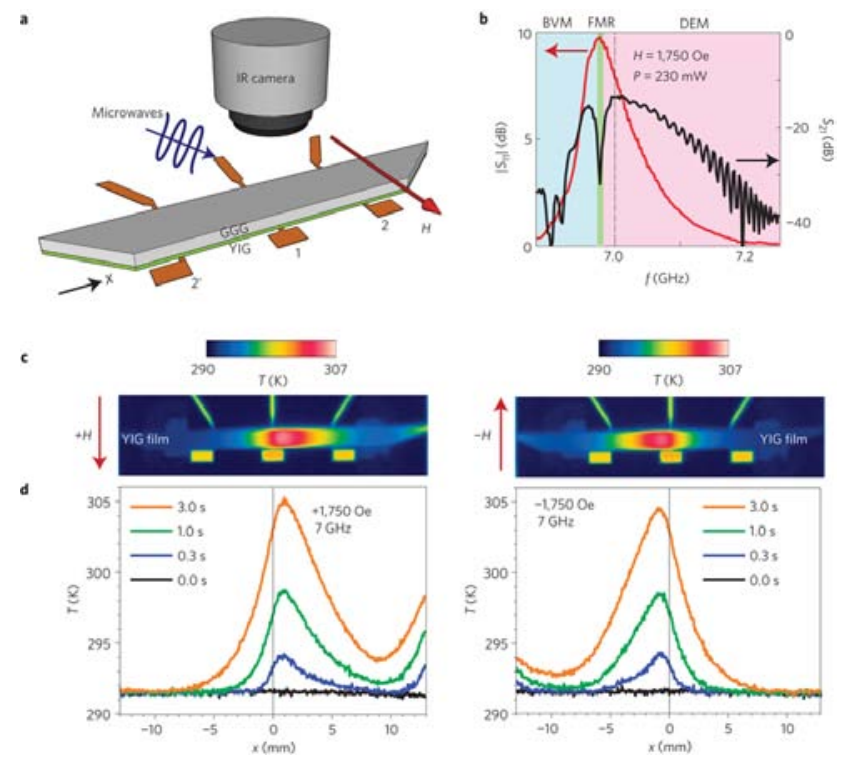

Figure 8: (a) Experimental set-up to detect heat generated from magnons excited in yttrium iron garnet with microwave antennas. (b) spin wave spectra measured from microwave absorption and transmission. (c),(d) Temperature profile and its temporal evolution for two opposite magnetic field polarities. Reprinted figure with permission from: Ref. [97] (c) 2013 Macmillan Publishers Ltd: Nature Materials.

concomitant increase in non-linear damping [95]. In contrast, by confining the current through the bilayer to a small region [as schematically show in Fig. 7(e)] a clear auto-oscillation signal is observed for sufficiently large $d c$ currents [89, 91], with non-linear dependence of the frequency, line-width and overall power, see Figs. 7(a)-(d). The excited magnetization mode consists of a self-localized standing spin wave ("bullet") soliton, which is enabled by propagating spin waves being damped due to radiation losses, i.e., traveling out of the excitation volume. Similarly, very complex dynamic excitations arise in more conventional spin-transfer torque nanocontacts, such as dissipative magnetic droplet solitons [96]. This demonstrates that careful geometric tuning of the available phase space for possible spin wave modes is important for understanding the complicated interplay between magnetization dynamics and spin currents.

\subsection{Heat current}

Like any other conducting material, metallic ferromagnets show thermoelectric effects with voltages generated from heat currents. For some of these, e.g., the Nernst effect, which is the thermal equivalent to Hall effects, their magnetic field dependence has been well known for a long time. More recently it was also shown that there are spin dependencies to the Seebeck (longitudinal voltage generated from heat currents) [98] and Peltier (temperature change generated by charge flow) [99] effects. While these effects can be understood in terms of the spin-dependent transport properties of the electrons, there have been related observations of so-called spin Seebeck [4] and spin Peltier [100] effects with ferromagnetic insulators, where charge transport is negligible. This indicates that in magnetically ordered systems heat transport by magnons can also play 
an important role. This in turn gave rise to a new direction of research called spin caloritronics, which explores the intersection between charge and heat transport with spin dynamics in magnetic structures and devices [101].

Fundamental to the understanding of spin caloritronic effects is the connection between magnon excitations and heat flow. The non-trivial nature of their interaction was demonstrated in a recent work by An et al. [97]. Using an infrared camera they detected the heat generated in an yttrium iron garnet (YIG) magnetic waveguide upon microwave excitation of socalled Damon-Eschbach spin wave modes, see Fig. 8(a). These are surface spin wave modes with a non-reciprocal propagation direction, which reverses upon magnetization reversal. As can be seen in Fig. 8(c) and (d) the heat is generated asymmetrically around the exciting microwave antenna, indicating that the magnons carry energy away from the place of microwave absorption before it is ultimately transferred as heat to lattice. Conversely, Costache et al. have shown that magnon heat transport can result in the generation of thermoelectric voltages in conducting ferromagnets [102]. Elucidating this interplay between heat transport, charge dynamics, magnons, and phonons is still one of the core challenges for a complete understanding of spin caloritronic effects, but at the same time may allow the development of new concepts for heat management with spin currents [103]. Conversely, heat current driven effects may enable novel approaches for characterizing magnetic properties [104, 105].

\subsection{Mechanical}

A century ago the seminal work by Einstein and de Haas (showing that a change in magnetization results in mechanical rotation) [106] and Barnett (showing that mechanical rotation results in a magnetization change) [107] proved unambiguously that magnetic moments also possess corresponding mechanical angular momentum. This fact has gained renewed interest, since several experiments have recently shown that this angular momentum related to magnetism is large enough to significantly modify the dynamics in microelectromechanical systems (MEMS)[108, 109, 110]. One example is shown in Fig. 9, which shows a MEMS torsional oscillator containing in the middle of the torsional bar a junction between a cobalt and gold wire. Passing a current from the cobalt into the gold will inject spin polarized electrons, which relax their polarization completely within the junction area due to the relatively short spin diffusion length in gold [111]. Using an $a c$ current for the spin injection will thus result in an oscillating torque, which can be detected by a change of the oscillation amplitude compared to a device with just a single cobalt wire. The analysis of the observed amplitude change yields a current polarization at the cobalt/gold interface of 0.85 , which is in line with other experimental measurements [109]. This observation inspired several different theoretical proposals for harnessing magnetomechanical coupling in nanoscale devices $[112,113]$, and many opportunities remain to explore the flow of angular mechanical momentum due to spin currents at the mesoscale. At the same time integrating magneto-mechanical effects into MEMS

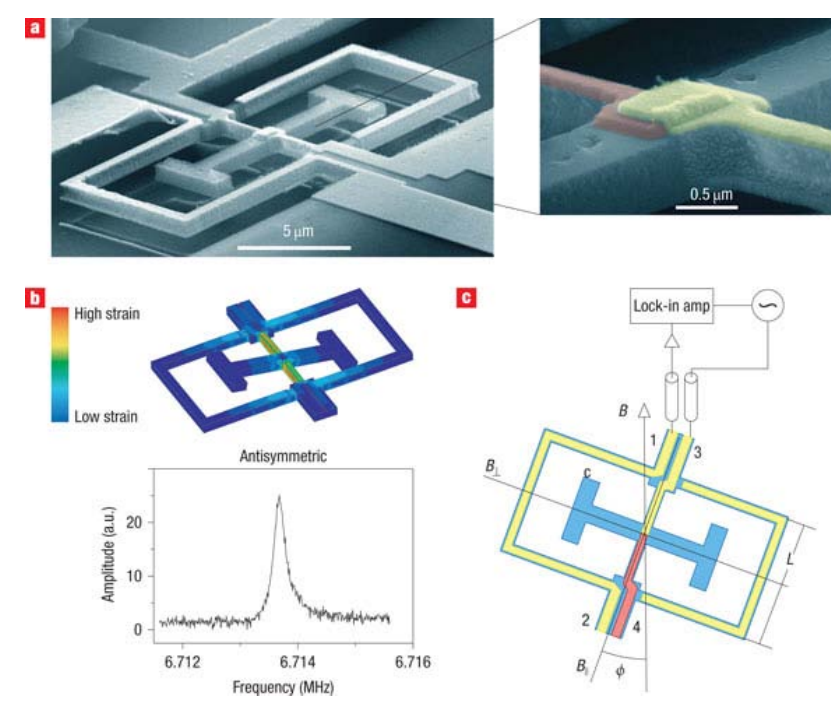

Figure 9: MEMS torsional oscillator for spin detection (a) Scanning electron microscopy image with cobalt wire colored in red and gold wire colored in yellow. (b) Finite element simulation of antisymmetric torsional mode and experimentally measured resonant signal. (c) Schematic of field orientation, which is used for polarizing cobalt, as well as generating a voltage from the motion on the outer frame. Reprinted figures with permission from: Ref. [109] (C) 2013 Macmillan Publishers Ltd: Nature Nanotechnolgy.

devices enables new concepts for energy scavenging from vibrations and thermal energies [114].

Beyond exploiting directly the connection between magnetic moments and mechanical torques, another possibility to harness the interaction between magnetic order and mechanical motion is through elastic strain coupling. Using this idea generated new approaches for magnetization switching in magnetostrictive materials coupled to piezoelectrics [115] or exciting ferromagnetic resonance with surface acoustic waves [116]. Furthermore, the idea of strain coupling is most pervasive in the quest for new multiferroic composites [117], which are envisioned as key components for sensors, actuators, microwave components, and possibly low dissipation information technologies.

\section{Conclusion}

The examples presented in this brief review show that mesoscale magnetism is a vibrant field of research that continuous to provide novel surprises. The complexity originating from the interplay of magnetic interactions over divergent length and time scales often produces a surprisingly rich variety of magnetic behavior that can be tailored via geometry or interactions between materials with different magnetic order. At the same time the coupling of the spin degree of freedom to charge, heat, and mechanical motion provides a wast phasespace for exploration, which undoubtedly will continue to yield novel phenomena. And if history provides any guidance, these progresses in the fundamental understanding of magnetic systems will continue to inspire novel application ranging from advanced information technologies to efficient energy conversion systems. 


\section{Acknowledgments}

Work at Argonne National Laboratory was supported by the U.S. Department of Energy, Office of Science, Materials Science and Engineering Division.

[1] B. Lambson, D. Carlton, J. Bokor, Exploring the thermodynamic limits of computation in integrated systems: Magnetic memory, nanomagnetic logic, and the Landauer limit, Phys. Rev. Lett. 107 (2011) 010604.

[2] M. Z. Hasan, J. E. Moore, Three-dimensional topological insulators, Annu. Rev. Condens. Matter Phys. 2 (2011) 55.

[3] J. M. Rondinelli, S. J. May, J. W. Freeland, Control of octahedral connectivity in perovskite oxide heterostructures: An emerging route to multifunctional materials discovery, MRS Bull. 37 (2012) 261.

[4] K. Uchida, J. Xiao, H. Adachi, J. Ohe, S. Takahashi, J. Ieda, T. Ota, Y. Kajiwara, H. Umezawa, H. Kawai, G. E. W. Bauer, S. Maekawa, E. Saitoh, Spin Seebeck insulator, Nat. Mater. 9 (2010) 894.

[5] I. M. Miron, K. Garello, G. Gaudin, P.-J. Zermatten, M. V. Costache, S. Auffret, S. Bandiera, B. Rodmacq, A. Schuhl, P. Gambardella, Perpendicular switching of a single ferromagnetic layer induced by in-plane current injection, Nature 476 (2011) 189.

[6] L. Liu, C.-F. Pai, Y. Li, H. W. Tseng, D. C. Ralph, R. A. Buhrman, Spintorque switching with the giant spin Hall effect of tantalum, Science 336 (2012) 555.

[7] L. H. Lewis, F. Jiménez-Villacorta, Perspectives on permanent magnetic materials for energy conversion and power generation, Metall. Mater. Trans. A 44 (2013) 2.

[8] E. F. Kneller, R. Hawig, The exchange-spring magnet: a new material principle for permanent magnets, IEEE Trans. Magn. 27 (1991) 3588.

[9] J. E. Davies, O. Hellwig, E. E. Fullerton, J. S. Jiang, S. D. Bader, G. T. Zimányi, K. Liu, Anisotropy dependence of irreversible switching in $\mathrm{Fe} / \mathrm{SmCo}$ and $\mathrm{FeNi} / \mathrm{FePt}$ exchange spring magnet films, Appl. Phys. Lett. 86 (2005) 262503.

[10] E. E. Fullerton, J. S. Jiang, M. Grimsdtich, C. H. Sowers, S. D. Bader, Exchange-spring behavior in epitaxial hard/soft magnetic bilayers, Phys. Rev. B 58 (1998) 12193.

[11] H. Zeng, J. Li, J. O. Liu, Z. L. Wang, S. Sun, Exchange-coupled nanocomposite magnets by nanoparticle self-assembly, Nature 420 (2002) 395.

[12] J. S. Jiang, S. D. Bader, Rational design of the exchange-spring permanent magnet, J. Phys.: Condens. Matter 26 (2014) 064212.

[13] H.-B. Braun, Topological effects in nanomagnetism: from superparamagnetism to chiral quantum solitons, Adv. Phys. 61 (2012) 1.

[14] 1. Gan, S. H. Chung, K. H. Aschenbach, M. Dreyer, R. D. Gomez, Pulsed-current-induced domain wall propagation in permalloy patterns observed using magnetic force microscope, IEEE Trans. Magn. 36 (2000) 3047.

[15] S. S. P. Parkin, M. Hayashi, L. Thomas, Magnetic domain-wall racetrack memory, Science 320 (2008) 190.

[16] F.-U. Stein, L. Bocklage, M. Weigand, G. Meier, Direct observation of internal vortex domain-wall dynamics, Phys. Rev. B 89 (2014) 024423.

[17] R. P. Cowburn, D. K. Koltsov, A. O. Adeyeye, M. E. Welland, D. M. Tricker, Single-domain circular nanomagnets, Phys. Rev. Lett. 83 (1999) 1042 .

[18] T. Shinjo, T. Okuno, R. Hassdorf, K. Shigeto, T. Ono, Magnetic vortex core observation in circular dots of permalloy, Science 289 (2000) 930.

[19] A. Wachowiak, J. Wiebe, M. Bode, O. Pietsch, M. Morgenstern, R. Wiesendanger, Direct observation of internal spin structure of magnetic vortex cores, Science 298 (2002) 577.

[20] R. K. Dumas, C. P. Li, I. V. Roshchin, I. K. Schuller, K. Liu, Magnetic fingerprints of sub-100 nm Fe dots, Phys. Rev. B 75 (2007) 134405.

[21] J. Sort, K. S. Buchanan, V. Novosad, A. Hoffmann, G. Salazar-Alvarez, A. Bollero, M. D. Baró, B. Dieny, J. Nogués, Imprinting vortices into antiferromagnets, Phys. Rev. Lett. 97 (2006) 067201.

[22] J. Wu, D. Carlton, J. S. Park, Y. Meng, E. Arenholz, A. Doran, A. T. Young, A. Scholl, C. Hwang, H. W. Zhao, J. Bokor, Z. Q. Qiu, Direct observation of imprinted antiferromagnetic vortex states in $\mathrm{CoO} / \mathrm{Fe} / \mathrm{Ag}(001)$ discs, Nat. Phys. 7 (2011) 303.

[23] S.-B. Choe, Y. Acremann, A. Scholl, A. Bauer, A. Doran, J. Stöhr, H. A. Padmore, Vortex core-driven magnetization dynamics, Science 304 (2004) 420 .
[24] V. Novosad, F. Y. Fradin, P. E. Roy, K. S. Buchanan, K. Yu. Guslienko, S. D. Bader, Magnetic vortex resonance in patterned ferromagnetic dots, Phys. Rev. B 72 (2005) 024455.

[25] K. S. Buchanan, P. E. Roy, M. Grimsdtich, F. Y. Fradin, K. Yu. Guslienko, S. D. Bader, V. Novosad, Soliton-pair dynamics in patterned ferromagnetic ellipses, Nat. Phys. 1 (2005) 172.

[26] S. Jain, V. Novosad, F. Y. Fradin, J. E. Pearson, V. Tiberkevich, A. N. Slavin, S. D. Bader, From chaos to selective ordering of vortex cores in interacting mesomagnets, Nat. Commun. 3 (2012) 1330.

[27] R. P. Cowburn, M. E. Welland, Room temperature magnetic quantum cellular automata, Science 287 (2000) 1466.

[28] A. Imre, G. Csaba, L. Ji, A. Orlov, G. H. Bernstein, W. Porod, Majority logic gate for magnetic quantum-dot cellular automata, Science 311 (2006) 205.

[29] D. A. Gilbert, G. T. Zimanyi, R. K. Dumas, M. Winkelhofer, A. Gomez, N. Elbagi, J. L. Vicent, K. Liu, Quantitative decoding of interactions in tunable nanomagnet arrays using first order reversal curves, Sci. Rep. 4 (2014) 4202

[30] K. Everschor-Sitte, M. Sitte, Real-space berry-phases: Skyrmion soccer (invited), J. Appl. Phys. 115 (2014) 172602

[31] A. Rosch, Skyrmions: Moving with the current, Nat. Nanotechn. 8 (2013) 160.

[32] A. Fert, V. Cros, J. Sampaio, Skyrmions on the track, Nat. Nanotechn. 8 (2013) 152.

[33] S. Mühlbauer, B. Binz, F. Jonietz, C. Pfleiderer, A. Rosch, A. Neubauer, R. Georgii, P. Böni, Skyrmion lattice in chiral magnet, Science 323 (2009) 915.

[34] D. Solenov, D. Mozyrsky, I. Martin, Chirality waves in two-dimensional magnets, Phys. Rev. Lett. 108 (2012) 096403.

[35] A. P. Malozemoff, J. C. Slonczewski, Magnetic Domain Walls in Bubble Materials, Academic Press, New York, 1979.

[36] X. Yu, M. Mostovoy, Y. Tokunaga, W. Zhang, K. Kimoto, Y. Matsui, Y. Kaneko, N. Nagaosa, Y. Tokura, Magnetic stripes and skyrmions with helicity reversals, Proc. Natl/ Acad, Sci. U.S.A. 109 (2012) 8856.

[37] S. Emori, U. Bauer, S.-M. Ahn, E. Martinez, G. S. D. Beach, Currentdriven dynamics of chiral ferromagnetic domain walls, Nat. Mater. 12 (2013) 611.

[38] K.-S. Ryu, L. Thomas, S.-H. Yang, S. Parkin, Chiral spin torque at magnetic domain walls, Nat. Nanotechn. 8 (2013) 527.

[39] A. Neubauer, C. Pfleiderer, B. Binz, A. Rosch, R. Ritz, P. G. Noklowitz, P. Böni, Topological Hall effect in the $A$ phase of MnSi, Phys. Rev. Lett. 102 (2009) 186602.

[40] S. Heinze, K. von Bergmann, M. Menzel, J. Brede, A. Kubetzka, R. Wiesendanger, G. Bihlmayer, S. Blügel, Spontaneous atomic-scale magnetic skyrmion lattice in two dimensions, Nat. Phys. 7 (2011) 713.

[41] T. Schultz, R. Ritz, A. Bauer, M. Halder, M. Wagner, C. Franz, C. Pfleiderer, K. Everschor, M. Garst, A. Rosch, Emergent electrodynamics of skyrmions in a chiral magnet, Nat. Phys. 8 (2012) 301.

[42] N. Romming, C. Hanneken, M. Menzel, J. E. Bickel, B. Wolter, K. von Bergmann, A. Kubetzka, R. Wiesendanger, Writing and deleting single magnetic skyrmions, Science 341 (2013) 636

[43] V. V. Kruglyak, S. O. Demokritov, D. Grundler, Magnonics, J. Phys. D Appl. Phys. 43 (2010) 264001.

[44] B. Lenk, H. Ulrichs, F. Garbs, M. Münzenberg, The building blocks of magnonics, Physics Reports 507 (2011) 107.

[45] A. A. Serga, A. V. Chumak, B. Hillebrands, YIG magnonics, J. Phys. D Appl. Phys. 43 (2010) 264002

[46] T. Schneider, A. Serga, B. Leven, B. Hillebrands, R. L. Stamps, M. Kostylev, Realization of spin-wave logic gates, Appl. Phys. Lett. 92 (2008) 022505.

[47] V. E. Demidov, S. Urazhdin, S. O. Demokritov, Control of spin-wave phase and wavelength by electric current on the microscopic scale, Applied Physics Letters 95 (2009) 262509.

[48] K. Vogt, H. Schultheiss, S. Jain, J. E. Pearson, A. Hoffmann, S. D. Bader, B. Hillebrands, Spin waves turning a corner, Appl. Phys. Lett. 101 (2012) 042410.

[49] T. Schneider, A. Serga, A. Chumak, C. Sandweg, S. Trudel, S. Wolff, M. Kostylev, V. Tiberkevich, A. Slavin, B. Hillebrands, Nondiffractive subwavelength wave beams in a medium with externally controlled anisotropy, Phys. Rev. Lett. 104 (2010) 197203.

[50] S. Mansfeld, J. Topp, K. Martens, J. Toedt, W. Hansen, D. Heitmann, 
S. Mendach, Spin wave diffraction and perfect imaging of a grating, Phys. Rev. Lett. 108 (2012) 047204.

[51] A. Chumak, P. Pirro, A. Serga, M. Kostylev, R. L. Stamps, H. Schultheiss, K. Vogt, S. Hermsdoerfer, B. Laegel, P. Beck, B. Hillebrands, Spin-wave propagation in a microstructured magnonic crystal, Appl. Phys. Lett. 95 (2009) 262508.

[52] H. Ulrichs, B. Lenk, M. Münzenberg, Magnonic spin-wave modes in CoFeB antidot lattices, Appl. Phys. Lett. 97 (2010) 092506.

[53] S. Tacchi, M. Madami, G. Gubbiotti, G. Carlotti, A. O. Adeyeye, S. Neusser, B. Botters, D. Grundler, Magnetic normal modes in squared antidot array with circular holes: A combined Brillouin light scattering and broadband ferromagnetic resonance study, IEEE Trans. Magn. 46 (2010) 172.

[54] B. Obry, P. Pirro, T. Brächer, A. V. Chumak, J. Osten, F. Ciubotaru, A. A. Serga, J. Fassbender, B. Hillebrands, A micro-structured ion-implanted magnonic crystal, Appl. Phys. Lett. 102 (2013) 202403.

[55] B. Obry, T. Meyer, P. Pirro, T. Brächer, B. Lägel, J. Osten, T. Strache, J. Fassbender, B. Hillebrands, Microscopic magnetic structuring of a spin-wave waveguide by ion implantation in a $\mathrm{Ni}_{81} \mathrm{Fe}_{19}$ layer, Appl. Phys. Lett. 102 (2013) 022409.

[56] S. O. Demokritov, A. A. Serga, V. E. Demidov, B. Hillebrands, M. Kostylev, B. Kalinikos, Experimental observation of symmetrybreaking nonlinear modes in an active ring, Nature 426 (2003) 159.

[57] M. Wu, B. Kalinikos, C. E. Patton, Self-generation of chaotic solitary spin wave pulses in magnetic film active feedback rings, Phys. Rev. Lett. 95 (2005) 237202.

[58] K. Vogt, F. Y. Fradin, J. E. Pearson, T. Sebastian, S. D. Bader, B. Hillebrands, A. Hoffmann, H. Schultheiss, Realization of a spin-wave multiplexer, Nat. Commun. 5 (2014) 3727.

[59] B. Hillebrands, J. Fassbender, Applied physics: Ultrafast magnetic switching, Nature 418 (2002) 493.

[60] J. Topp, D. Heitmann, M. P. Kostylev, D. Grundler, Making a reconfigurable artificial crystal by ordering bistable magnetic nanowires, Phys. Rev. Lett. 104 (2010) 207205.

[61] R. Hertel, W. Wulfhekel, J. Kirschner, Domain-wall induced phase shifts in spin waves, Phys. Rev. Lett. 93 (2004) 257202.

[62] C. Bayer, H. Schultheiss, B. Hillebrands, R. L. Stamps, Phase shift of spin waves traveling through a 180 degrees Bloch-domain wall, IEEE Trans. Magn. 41 (2005) 3094.

[63] D.-S. Han, S.-K. Kim, J.-Y. Lee, S. Hermsdoerfer, H. Schultheiss, B. Leven, B. Hillebrands, Magnetic domain-wall motion by propagating spin waves, Appl. Phys. Lett. 94 (2009) 112502.

[64] M. Kammerer, M. Weigand, M. Curcic, M. Noske, M. Sproll, A. Vansteenkiste, B. Van Waeyenberge, H. Stoll, G. Woltersdorf, C. H. Back, G. Schuetz, Magnetic vortex core reversal by excitation of spin waves, Nat. Commun. 2 (2011) 279.

[65] J. Iwasaki, A. J. Beekman, N. Nagaosa, Theory of magnon-kyrmion scattering in chiral magnets, Phys. Rev. B 89 (2014) 064412.

[66] E. Beaurepaire, J. C. Merle, A. Daunois, J.-Y. Bigot, Ultrafast spin dynamics in ferromagnetic nickel, Phys. Rev. Lett. 76 (1996) 4250.

[67] M. Djordjevic, M. Lüttich, P. Moschkau, P. Guderian, T. Kampfrath, R. G. Ulbrich, M. Münzenberg, W. Felsch, J. S. Moodera, Comprehensive view on ultrafast dynamics of ferromagnetic films, Phys. Stat. Solidi (c) 3 (2006) 1347

[68] B. Koopmans, G. Malinowski, F. Dalla Longa, D. Steiauf, M. Fähnle, T. Roth, M. Cinchetti, M. Aeschlimann, Explaining the paradoxical diversity of ultrafast laser-induced demagnetization, Nature Mater. 9 (2009) 259

[69] D. Rudolf, C. La-O-Vorakiat, M. Battiato, R. Adam, J. M. Shaw, E. Turgut, P. Maldonado, S. Mathias, P. Grychtol, H. T. Nembach, T. J. Silva, M. Aeschlimann, H. C. Kapteyn, M. M. Murnane, C. M. Schneider, P. M. Oppeneer, Ultrafast magnetization enhancement in metallic multilayers driven by superdiffusive spin current, Nat. Commun. 3 (2012) 1037

[70] T. Kampfrath, M. Battiato, P. Maldonado, G. Eilers, J. Nötzold, S. Mährlein, V. Zbarsky, F. Freimuth, Y. Mokrousov, S. Blugel, M. Wolf, I. Radu, P. M. Oppeneer, M. Münzenberg, Terahertz spin current pulses controlled by magnetic heterostructures, Nat. Nanotechn. 8 (2013) 256.

[71] M. van Kampen, C. Jozsa, J. Kohlhepp, P. LeClair, L. Lagae, W. de Jonge, B. Koopmans, All-optical probe of coherent spin waves, Phys. Rev. Lett. 88 (2002) 227201.
[72] T. Satoh, Y. Terui, R. Moriya, B. A. Ivanov, K. Ando, E. Saitoh, T. Shimura, K. Kuroda, Directional control of spin-wave emission by spatially shaped light, Nat. Photonics 6 (2012) 662.

[73] C. D. Stanciu, F. Hansteen, A. V. Kimel, A. Kirilyuk, A. Tsukamoto, A. Itoh, Th. Rasing, All-optical magnetic recording with circularly polarized light, Phys. Rev. Lett. 99 (2007) 047601.

[74] S. Mangin, M. Gottwald, C.-H. Lambert, D. Steil, V. Uhliř, L. Pang, M. Hehn, S. Alebrand, M. Cinchetti, G. Malinowski, Y. Fainman, M. Aeschlimann, E. E. Fullerton, Engineered materials for all-optical helicity-dependent magnetic switching, Nat. Mater. 13 (2014) 286.

[75] C.-H. Lambert, S. Mangin, B. S. D. Ch. S. Varaprasad, Y. K. Takahashi, M. Hehn, M. Cinchetti, G. Malinowski, K. Hono, Y. Fainman, M. Aeschlimann, E. E. Fullerton, All-optical control of ferromagnetic thin films and nanostructures, Science (2014), doi:10.1126/science. 1253493 .

[76] V. V. Temnov, G. Armelles, U. Woggon, D. Guzatov, A. Cebollada, A. Garcia-Martin, J.-M. Garcia-Martin, T. Thomay, A. Leitenstorfer, R. Bratschitsch, Active magneto-plasmonics in hybrid metalferromagnet structures, Nat. Photonics 4 (2010) 107.

[77] V. Bonanni, S. Bonetti, T. Pakizeh, Z. Pirzadeh, J. Chen, J. Nogués, P. Vavassori, R. Hillenbrand, J. Åkerman, A. Dmitriev, Designer magnetoplasmonics with nickel nanoferromagnets, Nano Lett. 11 (2012) 5333.

[78] J. C. Slonczewski, Current-driven excitation of magnetic multilayers, J. Magn. Magn. Mater. 159 (1996) L1.

[79] J. C. Slonczewski, Excitation of spin waves by an electric current, J. Magn. Magn. Mater. 195 (1996) L261.

[80] L. Berger, Emission of spin waves by a magnetic multilayer traversed by a current, Phys. Rev. B 54 (1996) 9353.

[81] J. A. Katine, F. J. Albert, R. A. Buhrman, E. B. Myers, D. C. Ralph, Current-driven magnetization reversal and spin-wave excitations in Co /Cu /Co pillars, Phys. Rev. Lett. 84 (2000) 3149.

[82] M. Tsoi, A. G. M. Jansen, J. Bass, W.-C.Chiang, V. Tsoi, P. Wyder, Generation and detection of phase-coherent current-driven magnons in magnetic multilayers, Nature 406 (2000) 46.

[83] S. I. Kiselev, J. C. Sankey, I. N. Krivorotov, N. C. Emley, R. J. Schoelkopf, R. A. Buhrman, D. C. Ralph, Microwave oscillations of a nanomagnet driven by a spin-polarized current, Nature 425 (2003) 380.

[84] M. I. D'yakonov, V. I. Perel', Possibility of orienting electron spins with current, Sov. Phys. JETP Lett. 13 (1971) 467.

[85] J. E. Hirsch, Spin Hall effect, Phys. Rev. Lett. 83 (1999) 1834.

[86] A. Hoffmann, Spin Hall effects in metals, IEEE Trans. Magn. 49 (2013) 5172.

[87] V. M. Edelstein, Spin polarization of conduction electrons induced by electric current in two-dimensional asymmetric electron systems, Solid State Commun. 73 (1990) 233.

[88] Y. Fan, P. Upadhyaya, X. Kou, M. Lang, S. Takei, Z. Wang, J. Tang, L. He, L.-T. Chang, M. Montazeri, G. Yu, W. Jiang, T. Nie, R. N. Schwartz, Y. Tserkovnyak, K. L. Wang, Magnetization switching through giant spin-orbit torque in a magnetically doped topological insulator heterostructure, Nat. Mater. 13 (2014) 699.

[89] V. E. Demidov, S. Urazhdin, H. Ulrichs, V. Tiberkevich, A. Slavin, D. Baither, G. Schmitz, S. O. Demokritov, Magnetic nano-oscillator driven by pure spin current, Nat. Mater. 11 (2012) 1028.

[90] L. Liu, C.-F. Pai, D. C. Ralph, R. A. Buhrman, Magnetic oscillations driven by the spin Hall effect in 3-terminal magnetic tunnel junction devices, Phys. Rev. Lett. 109 (2012) 186602.

[91] R. H. Liu, W. L. Lim, S. Urazhdin, Spectral characteristics of the microwave emission by the spin Hall nano-oscillator, Phys. Rev. Lett. 110 (2013) 147601

[92] Y. Kajiwara, K. Harii, S. Takahashi, J. Ohe, K. Uchida, M. Mizuguchi, H. Umezawa, H. Kawai, K. Ando, K. Takanashi, S. Maekawa, E. Saitoh, Transmission of electrical signals by spin-wave interconversion in a magnetic insulator, Nature 464 (2010) 262

[93] Z. Wang, Y. Sun, Y.-Y. Song, M. Wu, H. Schultheiß, J. E. Pearson, A. Hoffmann, Electric control of magnetization relaxation in thin film magnetic insulators, Appl. Phys. Lett. 99 (2011) 162511.

[94] A. Hoffmann, Make your spins spin, Physics 6 (2013) 39.

[95] V. E. Demidov, S. Urazhdin, E. R. J. Edwards, M. D. Stiles, R. D. McMichael, S. O. Demokritov, Control of magnetic fluctuations by spin current, Phys. Rev. Lett. 107 (2011) 107204.

[96] S. M. Mohensi, S. R. Sani, J. Persson, T. N. A. Nguyen, S. Chung, 
Ye. Pogoryelov, P. K. Muduli, E. Iacocca, A. Eklund, R. K. Dumas, S. Bonetti, A. Deac, M. A. Hoefer, J. Åkerman, Spin torque-generated magnetic droplet solitons, Science 339 (2013) 1295.

[97] T. An, V. I. Vasyuchka, K. Uchida, A. V. Chumak, K. Yamaguchi, K. Harii, J. Ohe, M. B. Jungfleisch, Y. kajiwara, H. Adachi, B. hillebrands, S. Maekawa, E. Saitoh, Unidirectional spin-wave heat conveyer, Nat. Mater. 12 (6) (2013) 549-553.

[98] A. Slachter, F. L. Bakker, J. P. Adam, B. J. van Wees, Thermally driven spin injection from a ferromagnet into a non-magnetic metal, Nat. Phys. 6 (2010) 879.

[99] J. Flipse, F. L. Bakker, A. Slachter, F. K. Dejene, B. J. van Wees, Direct observation of the spin-dependent Peltier effect, Nat. Nanotechn. 7 (2012) 166.

[100] J. Flipse, F. K. Dejene, D. Wagenaar, G. E. W. Bauer, J. B. Youssef, B. J. van Wees, Observation of the spin Peltier effect for magnetic insulators, Phys. Rev. Lett. 113 (2014) 027601.

[101] G. E. W. Bauer, E. Saitoh, B. J. van Wees, Spin caloritronics, Nat. Nanotechn. 11 (2012) 391.

[102] M. V. Costache, G. Bridoux, I. Neumann, S. O. Valenzuela, Magnondrag thermopile, Nat. Mater. 11 (2011) 199.

[103] J. Brüggemann, S. Weiss, P. Nalbach, M. Thorwart, Cooling a magnetic nanoisland by spin-polarized currents, Phys. Rev. Lett. 113 (2014) 076602.

[104] M. Weiler, M. Althammer, F. D. Czeschka, H. Huebl, M. S. Wagner, M. Opel, I.-M. Imort, G. Reiss, A. Thomas, R. Gross, S. T. B. Goennenwein, Local charge and spin currents in magnetothermal landscapes, Phys. Rev. Lett. 108 (2012) 106602.

[105] H. Schultheiss, J. E. Pearson, S. D. Bader, A. Hoffmann, Thermoelectric detection of spin waves, Phys. Rev. Lett. 109 (2012) 237204.

[106] A. Einstein, W. J. de Haas, Experimenteller Nachweis der Ampèreschen Molekularströme, Verh. Dtsch. Phys. Ges. 17 (1915) 152.

[107] S. J. Barnett, Magnetization by rotation, Phys. Rev. 6 (1915) 239.

[108] T. M. Wallis, J. Moreland, P. Kabos, Einstein-de Haas effect in a NiFe film deposited on a microcantilever, Appl. Phys. Lett. 89 (2006) 122502.

[109] G. Zolfagharkhani, A. Galdarzhy, P. Degiovanni, S. Kettemann, P. Fulde, P. Mohanty, Nanomechanical detection of itinerant electron spin flip, Nat. Nanotechn. 3 (2008) 720.

[110] S.-H. Lim, A. Imtiaz, T. M. Wallis, S. Russek, P. Kabos, L. Cai, E. M. Chudnovsky, Magneto-mechanical investigation of spin dynamics in magnetic multilayers, Europhys. Lett. 105 (2014) 37009.

[111] Y. Ji, A. Hoffmann, J. S. Jiang, S. D. Bader, Spin injection, diffusion, and detection in lateral spin-valves, Appl. Phys. Lett. 85 (2004) 6218.

[112] G. E. W. Bauer, S. Bretzel, A. Brataas, Y. Tserkovnyak, Nanoscale magnetic heat pumps and engines, Phys. Rev. B 81 (2010) 024427.

[113] M. Matsuo, J. Ieda, E. Saitoh, S. Maekawa, Effects of mechanical rotation on spin currents, Phys. Rev. Lett. 106 (2011) 076601.

[114] D. P. Arnold, Review of microscale magnetic power generation, IEEE Trans. Magn. 43 (2007) 3940.

[115] V. Novosad, Y. Otani, A. Ohsawa, S. G. Kim, K. Fukamichi, J. Kolke, K. Matuyama, O. Kitakami, Y. Shimada, Novel magnetostrictive memory device, J. Appl. Phys. 87 (2000) 6400.

[116] M. Weiler, L. Dreher, C. Heeg, H. Huebl, R. Gross, M. S. Brandt, S. T. B. Goennenwein, Elastically driven ferromagnetic resonance in nickel thin films, Phys. Rev. Lett. 106 (2011) 117601.

[117] C.-W. Nan, M. I. Bichurin, S. Dong, D. Viehland, G. Srinivasan, Multiferroic magnetoelectric composites: Historical perspective, status, and future directions, J. Appl. Phys. 103 (2008) 031101. 